\title{
Using Selective Withdrawal to Coat Microparticles
}

\author{
Itai Cohen, ${ }^{1}$ Hui Li, ${ }^{2}$ James L. Hougland, ${ }^{2}$ Milan Mrksich, ${ }^{2}$ \\ Sidney R. Nagel ${ }^{1}$
}

\begin{abstract}
We report a method that uses the process of selective withdrawal of one fluid through a second immiscible fluid to coat small particles with polymer films. Fluid is withdrawn through a tube with its orifice slightly above a water-oil interface. Upon increasing the flow rate, there is a transition from a state where only oil is withdrawn to a state where the water, containing the particles to be coated and appropriate prepolymer reagents, is entrained in a thin spout along with the oil. The entrained particles eventually cause the spout interface to break, producing a thin coat of controllable thickness around each particle, which can be subsequently polymerized using chemical reagents, light, or heat. This method allows flexibility in the chemical composition and thickness of the conformal coatings.
\end{abstract}

Coated microparticles are important in a range of technologies, including solid-phase resins for the synthesis of combinatorial libraries (1), immobilized catalysts in chemical production (2), protective coatings for cell and tissue encapsulation $(3,4)$, and vehicles

${ }^{1}$ James Frank Institute and Department of Physics, 2Department of Chemistry, University of Chicago, Chicago, IL 60637, USA. for local drug delivery (5). Several coating methods are used because no single technique is best suited for all types of particles and polymerization schemes. Current options for encapsulation include droplet generation (6) or coacervation (7), emulsion formation (8), polyelectrolyte multilayering (9), and direct polymerization from a surface-adsorbed initiator (10). Many of these techniques (6-8) have the limitation that they produce particles
A

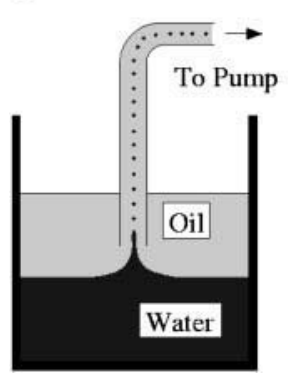

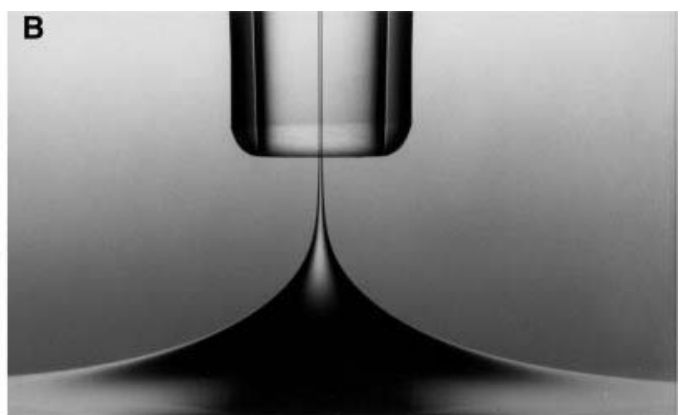

Fig. 1. (A) Diagram of the apparatus used in selective withdrawal. The tube orifice is a distance $S$ from the unperturbed interface, and the rate of total fluid withdrawal is $Q$. A gear pump (Tuthill D9005M) was used to withdraw the fluids through a tube with $S$ between 6.5 and $8.0 \mathrm{~mm}$. $Q$ was typically between 0.7 and $1.5 \mathrm{ml} / \mathrm{s}$. Siphoning can also be used. (B) Photograph of a water spout entrained with the heavy mineral oil in a tube with orifice diameter $1.6 \mathrm{~mm}$. The viscosities of the water and oil are $1.0 \mathrm{cSt}$ and $195 \mathrm{cSt}$, respectively, at room temperature. The surface tension of the water-oil interface is $35 \pm 3$ dynes $/ \mathrm{cm}$.

Fig. 2. Photograph of a poppy seed inside the water spout. The spout stretches around the particle and eventually breaks up both above and below the particle to produce particles encapsulated in an aqueous shell of uniform thickness. The orifice diameter is $\mathbf{1 . 2}$ $\mathrm{mm}$ and the particle diameter is about $0.8 \mathrm{~mm}$.

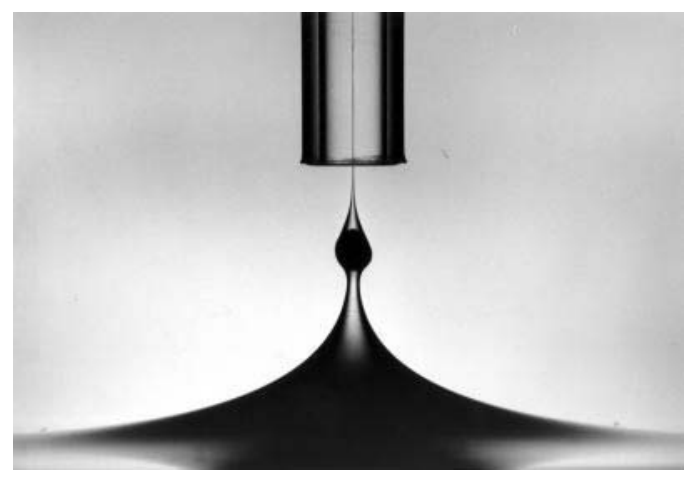

of a constant total size, including the coat, even when the uncoated particles have a heterogeneous size distribution. Other coating techniques $(9,10)$ are able to produce coats of uniform thickness, but may often be restricted with respect to the chemical composition of the coat and the polymerization schemes that can be used. There remains an important need for a versatile coating technology that allows for the use of a variety of polymerization schemes and can apply uniform coats of diverse composition and controllable thickness onto polydisperse particles.

We report a method that uses the process of selective withdrawal $(11,12)$ to apply polymer coats of uniform thickness and varying composition onto polydisperse particles. In the simplest version of this experiment, a tube is introduced into a container such that its tip is suspended at a height $S$ above an interface separating two immiscible liquids (Fig. 1A). For low rates of fluid withdrawal $Q$, only the upper fluid, which in this experiment is oil, is withdrawn through the tube. An increase in $Q$ (or a decrease in $S$ ) leads to a transition where the lower liquid (which in this experiment is water) is entrained in a thin spout along with the oil (Fig. 1B) (12). Once the spout has formed, an increase in $Q$ or a reduction in $S$ causes the spout to thicken. A higher oil viscosity produces thinner initial spouts (13).

When particles are added to the aqueous phase, they become entrained in the spout. Because the spout gets progressively thinner as it rises above the interface, it eventually becomes thinner than the entrained particle, which causes the interface to stretch (Fig. 2) and eventually break both above and below the particle, leaving a thin water shell surrounding the particle. A key feature of the spout collapse is that larger particles break up the spout earlier than smaller ones, and so the thickness of the shell is relatively independent of the particle size. The viscous outer fluid (14) suppresses flow instabilities in the

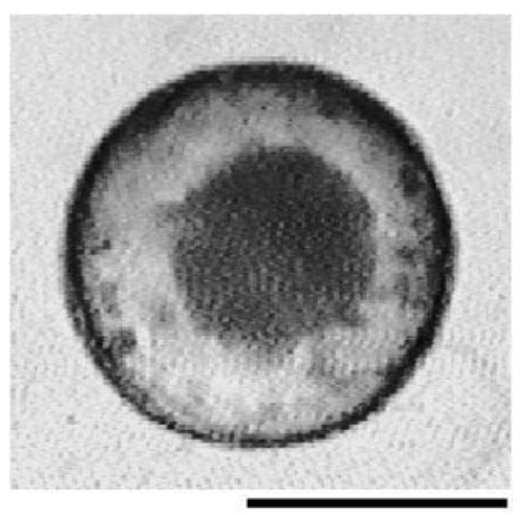

Fig. 3. Optical micrograph of a corn pollen particle encapsulated with a polyamide coat. The corn pollen appears as a dark, solid elliptical object $83 \mu \mathrm{m}$ in diameter; the coat is about $38 \mu \mathrm{m}$ thick. Scale bar, $100 \mu \mathrm{m}$. 
R E P O R T S

Fig. 4. Plot of coat thickness versus particle diameter. The flat distribution indicates that coat thickness does not change with particle size. The inset shows a histogram distribution of coat thickness for the same run in which both $Q$ and $S$ are held constant at $1.5 \mathrm{ml} / \mathrm{s}$ and $8.0 \mathrm{~mm}$, respectively. The distribution can be fit with a Gaussian and has a standard deviation of $4 \mu \mathrm{m}$.

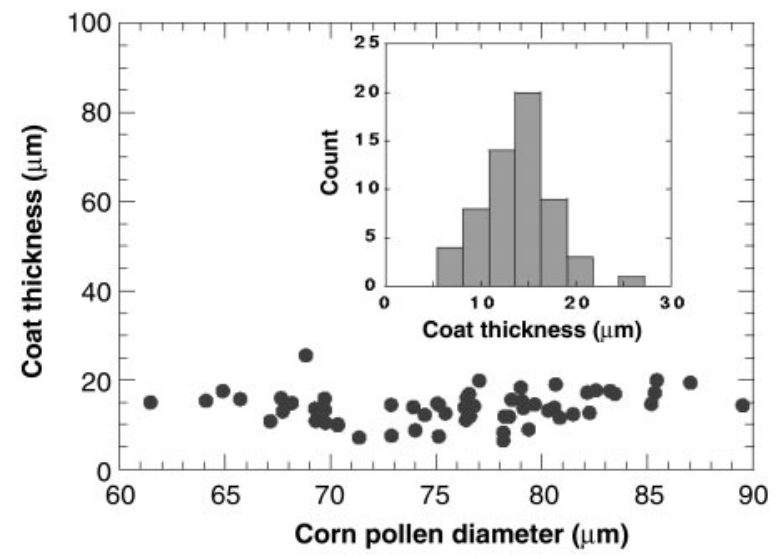

than that shown in Fig. 3.

We used confocal and optical microscopies cess can be adopted to apply polymer coatings onto the particles by adding reagents to the water that can subsequently be crosslinked (15). As with capillary flow focusing (16), selective withdrawal prevents tip clogging (17) by never allowing the prepolymer spout to touch the tube.

We now describe one example of applying a polyamide coat by chemically crosslinking a low molecular weight poly(ethylene glycol). The lower phase consists of $\mathrm{D}_{2} \mathrm{O}$ containing $O, O^{\prime}$-bis(2-aminopropyl) poly(ethylene glycol), molecular weight 800 [5\% $\mathrm{w} / \mathrm{v}$, viscosity $1.4 \mathrm{cSt}$ (where $1 \mathrm{cSt}=10^{-2}$ $\mathrm{cm}^{2} \mathrm{~s}^{-1}$ at room temperature)] and a polydisperse suspension of corn pollen particles (average diameter $100 \mu \mathrm{m}$ ). The higher density of $\mathrm{D}_{2} \mathrm{O}$ relative to $\mathrm{H}_{2} \mathrm{O}$ keeps the corn pollen particles near the interface, where they efficiently enter the spout. The encapsulated particles are then deposited in a separate bath of heavy mineral oil containing 1,3,5-benzenetricarbonyl trichloride (15 mM). Upon contact with the aqueous film, the reagent reacts with the amino groups of the polyethylene glycol to produce a cross-linked coat around the particle (Fig. 3). With this scheme, the particle is never exposed to the reagent that induces polymerization. As described below, the coat can be made much thinner or thicker

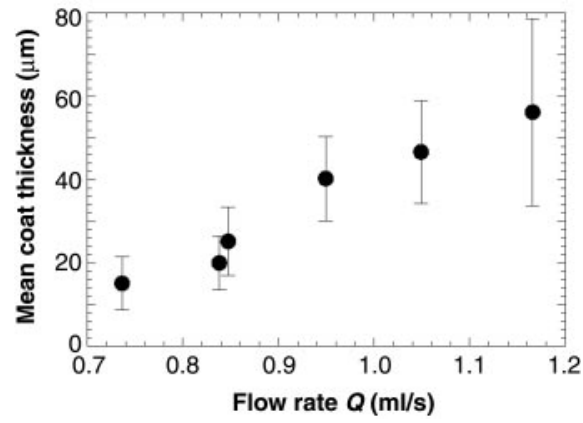

Fig. 5. Plot of mean coat thickness as a function of $Q$, for $S=6.5 \mathrm{~mm}$. The mean thickness increases linearly with $Q$; error bars represent standard deviation of thickness.
$6.5 \mathrm{~mm}$. For flow rates of $0.74 \mathrm{ml} / \mathrm{s}$, the corn pollen coats had a thickness of $15 \pm 5 \mu \mathrm{m}$. Increasing $Q$ produces thicker coats, consistent with the trend that the spout becomes thicker at higher $Q$. The error bars, corresponding to the standard deviations of the distributions, increase with $Q$. The width is due in part to drop coalescence and should decrease when fast cross-linking schemes are initiated immediately after coat formation. Such rapid cross-linking can also be used to counteract buoyancy effects that occasionally yield off-center particles.

In contrast to the current droplet generation techniques $(6-8)$, selective withdrawal ensures that all particles receive a coat of equal mean thickness (Fig. 4), and this thickness can be controlled by adjusting the experimental parameters (for example, by varying $Q$ as shown in Fig. 5). This might be useful in potential future applications involving the coating of transplanted biological cells in order to protect them from the host immune system $(3,4)$. For example, the irregular shape and size of cell clusters (such as islet cells transplanted to regulate the delivery of insulin into the bloodstream) makes it difficult to produce coats that are uniform in thickness.

We have described one possible scheme that uses selective withdrawal to encapsulate

a particle and a chemical initiator to subse-

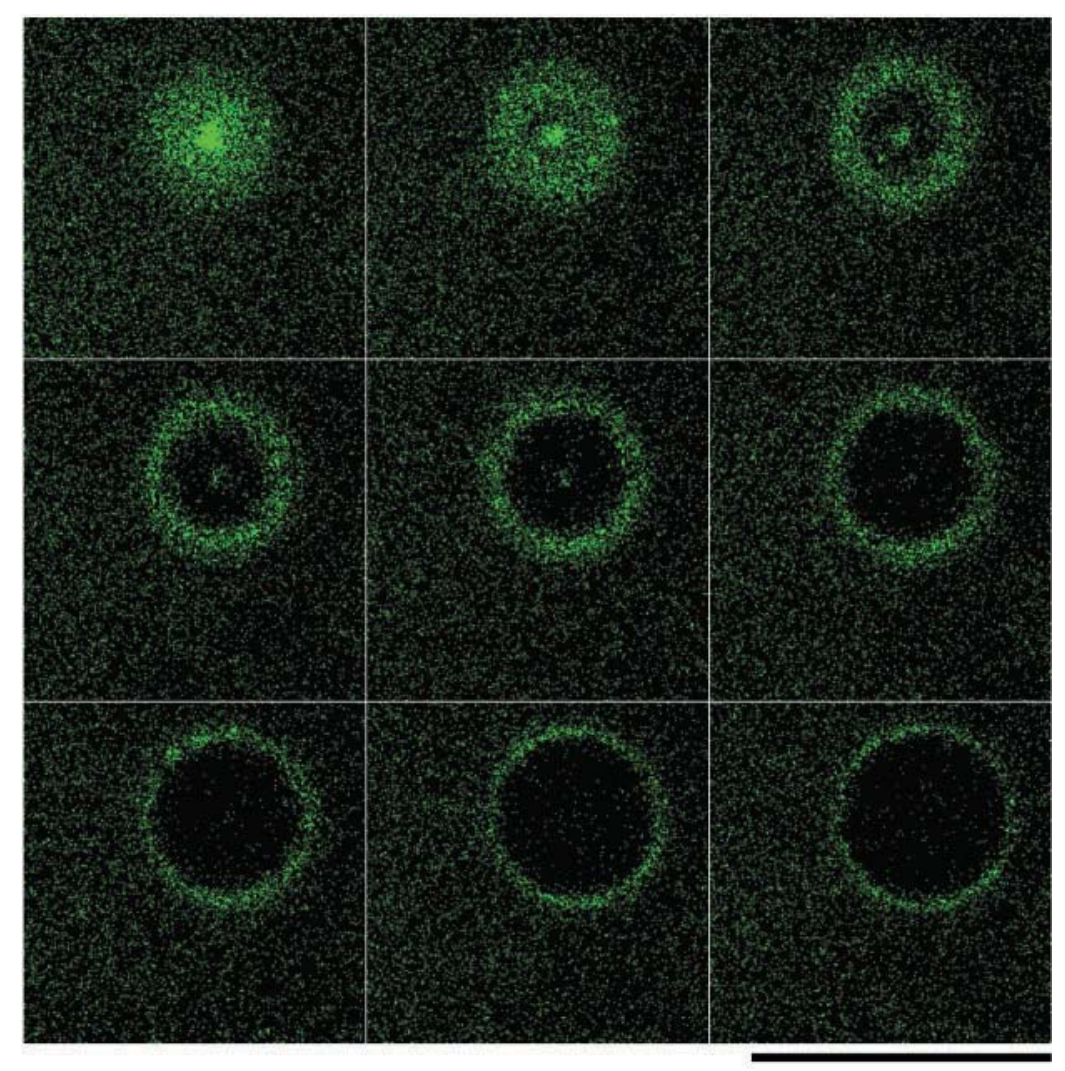

Fig. 6. Confocal images of a polystyrene bead coated with agarose (type XII, Sigma). After selective withdrawal, the coat was hardened by cooling the coat-plus-particle systems to temperatures lower than the gelling point of the agarose. The agarose contains PolyFluor 512 (Polysciences), which fluoresces while the polystyrene bead, $220 \mu \mathrm{m}$ in diameter, does not fluoresce. Successive cross-sectional image planes show that the agarose coat has a uniform thickness of $12 \mu \mathrm{m}$. The agarose rings increase in diameter with cross sections nearing the particle's equatorial plane. Beyond this point, the particle itself starts to block the light. Scale bar, $0.4 \mathrm{~mm}$. 
quently harden the coat. However, this coating method is easily generalized to allow for different ways of hardening the particle coats. With the use of appropriate reagents, this technique is also compatible with schemes that thermally or optically initiate the hardening of the coats. Figure 6 shows confocal images of a polystyrene bead encapsulated with an agarose coat that was hardened by lowering its temperature. In Fig. 7, we show an optical micrograph of a poppy seed encapsulated in a poly(styrenesulfonic acid) coat that was photopolymerized. The selective withdrawal geometry may also be inverted by inserting the straw through the bottom of the withdrawal container, with the straw tip positioned below the interface. Now the denser aqueous fluid is the primary fluid being withdrawn, and the particles to be coated are placed in the oil (upper fluid). This inversion extends the applicability of this coating technique to hydrophobic particles in oil-soluble reagents as well as to heavy particles in the upper fluid that will sediment to the interfacial boundary.

The selective withdrawal coating technique complements other currently available techniques, such as surface-induced polymerization (10), which can also produce coats of uniform thickness on irregularly shaped particles but which often require modification of the particle surface and therefore may not be feasible. In our technique, as illustrated in the description of the polyamide coating, the particles can be completely separated from the caustic reagents that initiate the polymerization at the outer interface. Moreover, surfaceinduced polymerization requires the reagent to be stable in the solution being polymer-

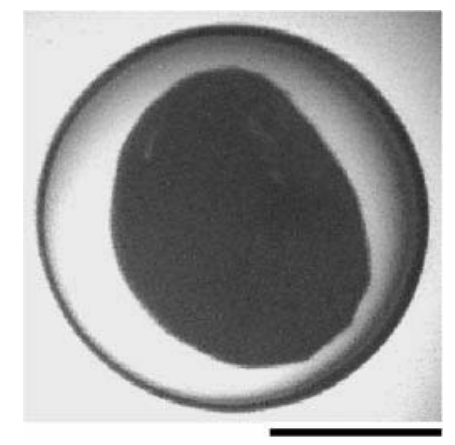

Fig. 7. Optical micrograph of a poppy seed encapsulated in a poly(styrenesulfonic acid) coat. The poppy seed appears as a dark solid object $0.9 \mathrm{~mm}$ in diameter; the coat is $0.2 \mathrm{~mm}$ thick. The prepolymer used is styrene sulfonic acid sodium salt $\left(30 \% \mathrm{w} / \mathrm{v}\right.$ in $\left.\mathrm{D}_{2} \mathrm{O}\right)$ and triethylene glycol diacrylate $\left(5 \% \mathrm{w} / \mathrm{v}\right.$ in $\left.\mathrm{D}_{2} \mathrm{O}\right)$ mixed with eosin $Y(0.5 \mathrm{mM})$ and triethanolamine $(100 \mathrm{mM})$ as the photosensitizer-electron donor initiating system (18). After the selective withdrawal process, the coated particles were collected in a plastic container and irradiated for $20 \mathrm{~min}$ with a halogen lamp. Scale bar, 0.5 $\mathrm{mm}$. ized. This is not always possible (as with the polyamide coat installation, the trichloride reagent is unstable in aqueous solutions), and selective withdrawal can solve this problem by initiating polymerization with a reagent that is stable in the fluid surrounding the coat. Finally, many polymer coatings are either difficult [e.g., the photopolymerized poly(styrenesulfonic acid) coat shown in Fig. 7] or impossible (e.g., the thermally hardened agarose coat shown in Fig. 6) to prepare using techniques such as surface-induced polymerization and must be hardened in bulk. For these cases in particular, selective withdrawal presents a valuable advantage over currently available coating techniques.

The selective withdrawal technique can be readily optimized. With a single tube we estimate that 10,000 particles can be coated per hour. Preliminary experiments have demonstrated that this technique can be scaled up by using an array of tubes in parallel. Injecting particles directly into the region below the spout can make the method suitable for particles with higher density than the prepolymer. As described above, inversion of the selective withdrawal geometry can extend the applicability of this technique to hydrophobic particles in oilsoluble reagents. Because of its flexibility in polymerization schemes, its ability to coat particles of many different types, and its ability to tune the thickness of the coats, this technique is an attractive option in a range of applications and a valuable addi- tion to the repertoire of currently available coating techniques.

References and Notes

1. A. J. Mendonca, X. Y. Xiao, Med. Res. Rev. 19, 451 (1999).

2. S. J. Shuttleworth, S. M. Allin, P. K. Sharma, Synthesis 11, 1217 (1997).

3. F. Lim, A. M. Sun, Science 210, 908 (1980).

4. P. Soon-Shiong, Adv. Drug Deliv. Rev. 35, 259 (1999).

5. R. Langer, Acc. Chem. Res. 33, 94 (2000).

6. G. H. J. Wolters, W. M. Fritschy, D. Gerrits, R. Van Schilfgaarde, J. Appl. Biomater. 3, 281 (1992).

7. T. Yoshioka, R. Hirano, T. Shioya, M. Kako, Biotechnol. Bioeng. 35, 66 (1990).

8. E. Mathiowitz, Encyclopedia of Controlled Drug Delivery (Wiley, New York, 1999).

9. E. Donath, G. B. Sukhorukov, F. Caruso, S. A. Davis, H. Mohwald, Angew. Chem. Int. Ed. 37, 2202 (1998).

10. G. M. Cruise, O. D. Hegre, D. S. Scharp, J. A. Hubbell, Biotechnol. Bioeng. 57, 655 (1998).

11. J. R. Lister, J. Fluid Mech. 198, 231 (1989).

12. S. Blake and G. N. Ivey, J. Volcanol. Geotherm. Res. 27, 153 (1986).

13. I. Cohen and S. R. Nagel, in preparation.

14. E. E. Timm, U.S. Patent $4,444,961$ (1984).

15. Even without the inclusion of particles, as originally shown by Savart [Annal. Chim. 53, 337 (1883)] and Rayleigh [Philos. Mag. 34, 177 (1892)], the prepolymer spout will break into droplets that can be hardened, allowing the fabrication of monodisperse particles.

16. A. M. Ganan-Calvo, Phys. Rev. Lett. 80, 285 (1997).

17. P. B. Umbanhowar, V. Prasad, D. A. Weitz, Langmuir 16, 347 (2000)

18. O. Valges-Aguilera, C. P. Pathak, J. Shi, D. Watson, D. C. Neckers, Macromolecules 25, 541 (1992).

19. We thank H. Rilo and A. Rotamel for early discussions that motivated these studies. We also thank C. Lassy and the Confocal Digital Imaging Facility at the University of Chicago. Supported by NSF grant DMR9722646 and NSF Materials Research Science and Engineering Centers Program grant DMR-9808595.

19 January 2001; accepted 7 March 2001

\title{
Anthropogenic Warming of Earth's Climate System
}

\author{
Sydney Levitus, ${ }^{1 *}$ John I. Antonov, ${ }^{1}$ Julian Wang, ${ }^{2}$ \\ Thomas L. Delworth, ${ }^{3}$ Keith W. Dixon, ${ }^{3}$ Anthony J. Broccoli ${ }^{3}$
}

\begin{abstract}
We compared the temporal variability of the heat content of the world ocean, of the global atmosphere, and of components of Earth's cryosphere during the latter half of the 20th century. Each component has increased its heat content (the atmosphere and the ocean) or exhibited melting (the cryosphere). The estimated increase of observed global ocean heat content (over the depth range from 0 to 3000 meters) between the 1950s and 1990s is at least one order of magnitude larger than the increase in heat content of any other component. Simulation results using an atmosphere-ocean general circulation model that includes estimates of the radiative effects of observed temporal variations in greenhouse gases, sulfate aerosols, solar irradiance, and volcanic aerosols over the past century agree with our observation-based estimate of the increase in ocean heat content. The results we present suggest that the observed increase in ocean heat content may largely be due to the increase of anthropogenic gases in Earth's atmosphere.
\end{abstract}

Studies using instrumental data to document a warming of Earth's climate system due to increasing concentrations of greenhouse gases (GHGs) have focused on surface air temperature and sea surface temperature (1).
These variables have proved invaluable for documenting an average warming of approximately $0.6^{\circ} \mathrm{C}$ at Earth's surface (1) during the past 100 years. Recent comparisons (2-4) with paleoclimatic proxy data indicate that 